Research Article

\title{
Silver Chloride Encapsulation-Induced Modifications of Raman Modes of Metallicity-Sorted Semiconducting Single-Walled Carbon Nanotubes
}

\author{
M. V. Kharlamova ${ }^{D},{ }^{1}$ C. Kramberger, ${ }^{2}$ A. Mittelberger, ${ }^{2}$ K. Yanagi, ${ }^{3}$ T. Pichler, ${ }^{2}$ and D. Eder ${ }^{1}$ \\ ${ }^{1}$ Institute of Materials Chemistry, Vienna University of Technology, Getreidemarkt 9/BC/2, 1060 Vienna, Austria \\ ${ }^{2}$ Faculty of Physics, University of Vienna, Strudlhofgasse 4, 1090 Vienna, Austria \\ ${ }^{3}$ Department of Physics, Tokyo Metropolitan University, 1-1 Minami-Osawa, Hachioji-shi, Tokyo 192-0397, Japan
}

Correspondence should be addressed to M. V. Kharlamova; mv.kharlamova@gmail.com

Received 17 November 2017; Revised 17 January 2018; Accepted 29 January 2018; Published 1 April 2018

Academic Editor: Jau-Wern Chiou

Copyright ( 2018 M. V. Kharlamova et al. This is an open access article distributed under the Creative Commons Attribution License, which permits unrestricted use, distribution, and reproduction in any medium, provided the original work is properly cited.

\begin{abstract}
The internal channels of semiconducting single-walled carbon nanotubes (SWCNTs) were filled with silver chloride. The filling was confirmed by high-resolution scanning transmission electron microscopy. The filling-induced modifications of Raman modes of SWCNTs were analyzed. The fitting of the radial breathing mode (RBM) and G-bands of Raman spectra of the pristine and filled nanotubes with individual components allowed analyzing in detail the influence of encapsulated silver chloride on the electronic properties of different diameter nanotubes. The analysis of the RBM-band allowed revealing the changes in resonance excitation conditions of SWCNTs upon filling. The analysis of the G-band allowed concluding about $p$-doping of nanotubes by incorporated silver chloride accompanied by charge transfer from nanotubes to the inserted salt.
\end{abstract}

\section{Introduction}

The unique physical, chemical, and mechanical properties of single-walled carbon nanotubes (SWCNTs) made them an object of investigation in fundamental and applied science. The properties of SWCNTs depend solely on their atomic structure [1]. As-synthesized nanotubes represent a mixture of SWCNTs with metallic and semiconducting conductivity type, which limits their applications [2]. To control the properties of synthesized SWCNTs, two methods were established: (i) separation of nanotubes and (ii) chemical functionalization of SWCNTs [3].

The separation techniques allow obtaining high-purity SWCNTs with defined conductivity type, diameter, and even chiral angle [4]. Chemical functionalization methods allow modification of the electronic properties of SWCNTs. Among chemical functionalization methods, the filling of SWCNT channels was shown to be an effective way to obtain nanotubes with the required properties [3]. It was demonstrated that the filling of SWCNTs with simple elemental substances [5-7], chemical compounds [8-14], and molecules [15-19] allows tailoring the electronic properties of nanotubes by varying the doping type and level [3].

Raman spectroscopy is a powerful technique for the investigation of the electronic properties of carbon nanotubes $[20,21]$. The analysis of the radial breathing mode (RBM) allows determining the diameter distribution of nanotubes, whereas the analysis of the G-band allows revealing doping and hybridization effects. There are reports on the investigation of the filled SWCNTs by Raman spectroscopy [22-24]. However, all of them deal with mixed nanotube samples containing SWCNTs with metallic and semiconducting conductivity type. Moreover, these works used only few laser wavelengths, which makes it difficult to assess filling-induced changes in Raman modes of different nanotubes. There are no reports in the literature on a detailed investigation of modifications of Raman spectra of highly pure metallicitysorted SWCNTs upon filling. However, such an investigation is required for a comprehensive understanding of influence of filler materials on the electronic properties of SWCNTs. 
These data are needed to assess the application potential of filled nanotubes in nanoelectronics.

In this contribution, we close this gap by performing a detailed study of filling-induced alteration of Raman modes of high-purity semiconducting SWCNTs upon filling with silver chloride $(\mathrm{AgCl})$. The filling of nanotube channels is confirmed by high-resolution scanning transmission electron microscopy (HR STEM). The filled SWCNTs are investigated by multifrequency Raman spectroscopy at five laser wavelengths. Using different laser wavelengths allows exciting electronic transitions of different semiconducting nanotubes and investigating the influence of incorporated silver chloride on their electronic properties. The fitting of the RBM and G-bands of Raman spectra of the pristine and filled SWCNTs allows comparing peak positions and relative intensities of individual components and concluding about changes in resonance conditions and the electronic properties of SWCNTs upon filling. It is found that the inserted silver chloride causes $p$-doping of host nanotubes accompanied by charge transfer from nanotubes to the salt.

\section{Materials and Methods}

Semiconducting SWCNTs were obtained by density gradient ultracentrifugation of mixed SWCNT sample with a mean diameter of $1.4 \mathrm{~nm}$ produced by the arc-discharge method (ArcSO type, Meijo Nano Carbon Co.). The procedure of the separation of SWCNTs by conductivity type was described in $[25,26]$. Briefly, the dispersion of SWCNTs in $2 \%$ solution of deoxycholate sodium salt (DOC, Tokyo Chemical Industry Co.) was prepared using a digital sonifier. The solution of SWCNTs and five mixtures of $2 \%$ sodium dodecyl sulfate (SDS, Aldrich) solution with 27.5, 30, 32.5, 35 , and $40 \mathrm{wt} \%$ iodixanol (COSMO BIO Co.) solutions were put layer by layer into a centrifuge tube (Hitachi Koki Co.) in order to form the density gradient. After ultracentrifugation of the tube, semiconducting SWCNTs were extracted from its bottom part. The cleaning of SWCNTs from the surfactants and gradient media was performed through repetition of washing procedures using methanol, hot water, and toluene, as described in [25]. Films of purified semiconducting nanotubes were obtained. The high purity and quality of these samples were confirmed in [26].

Prior to filling, the films of semiconducting SWCNTs were annealed in air at $500^{\circ} \mathrm{C}$ for $15 \mathrm{~min}$ in order to open the nanotube ends. After that, the SWCNTs and silver chloride were placed in a quartz ampoule and sealed. The ampoule was heated in the tube furnace up to a temperature of $555^{\circ} \mathrm{C}$ and kept at this temperature for 6 hours. The ampoule was slowly then cooled to room temperature with a rate of $0.05-1.5^{\circ} \mathrm{C} / \mathrm{min}$.

The HR STEM imaging was conducted with a Nion UltraSTEM 100 microscope at an accelerating voltage of $60 \mathrm{kV}$. The samples were prepared by drop casting of the solution of filled SWCNTs in isopropanol on carbon-coated copper grids. The Raman spectroscopy measurements were conducted at a Horiba Jobin Yvon LabRAM HR800 spectrometer equipped with an external tunable $\mathrm{ArKr}$ laser (Coherent Innova 70c) allowing excitation wavelengths of

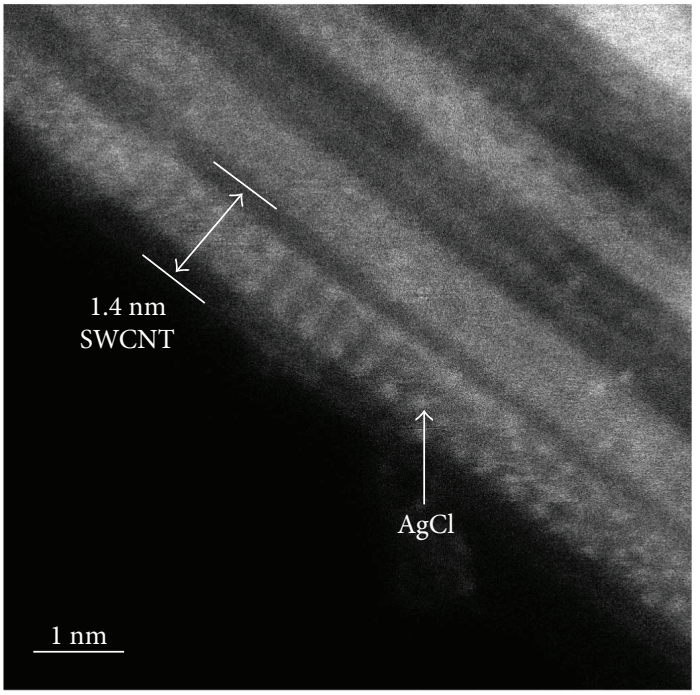

FIgURE 1: The HR STEM microphotograph of AgCl-filled SWCNTs.

$458,488,514,531$, and $568 \mathrm{~nm}$ or $2.71,2.54,2.41,2.34$, and $2.18 \mathrm{eV}$, respectively. The RBM- and G-bands of Raman spectra were fitted with Voigtian and Fano peaks, and area intensities were calculated in PeakFit v4.12. The accuracy in peak positions is $\pm 2 \mathrm{~cm}^{-1}$.

\section{Results and Discussion}

Figure 1 presents the HR STEM micrograph of AgCl-filled SWCNTs. It reveals contrast elements inside the nanotube channels, which corresponds to individual atoms of the encapsulated compound. It proves the successful filling of SWCNTs with silver chloride. The distances between atoms in the directions which are parallel and perpendicular to the nanotube axis amount to 0.43 and $0.52 \mathrm{~nm}$, respectively. The latter fits to the lattice parameter of bulk $\mathrm{AgCl}$ ( $a=0.546 \mathrm{~nm}, \mathrm{NaCl}$ structure, Fm $3 \mathrm{~m}$ space group) [27].

The Raman spectrum of SWCNTs includes four main features: (i) the radial breathing mode (RBM), which corresponds to radial vibrations of carbon atoms, (ii) the D-band, which is related to a symmetry forbidden zone boundary inplane mode of graphene sheets that is enabled by structural defects and disordering, (iii) the G-band, which corresponds to longitudinal and tangential vibrations of carbon atoms, and (iv) the 2D-band, which is related to symmetry allowed overtone of the D-line [20].

Figure 2 shows the RBM, D, G, and 2D-bands of Raman spectra of the pristine and AgCl-filled SWCNTs acquired at laser wavelengths of $458-568 \mathrm{~nm}$. The peaks of the RBMband are positioned at frequencies ranging between 150 and $190 \mathrm{~cm}^{-1}$. The peaks of D-band are placed between 1330 and $1360 \mathrm{~cm}^{-1}$. The peaks of G-band are located between 1550 and $1600 \mathrm{~cm}^{-1}$. The peaks of 2D-band are placed between 2660 and $2710 \mathrm{~cm}^{-1}$. The spectra of the pristine and filled nanotubes show noticeable differences in peak positions as well as peak profile (Figure 2). 

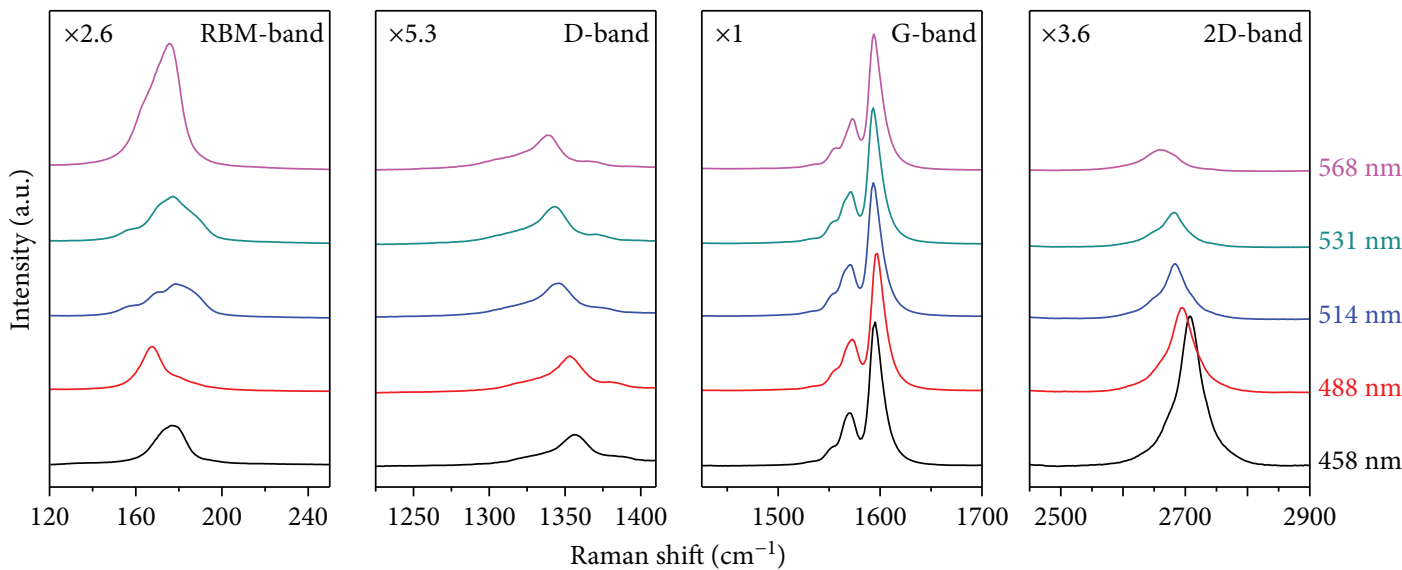

(a) SWCNT
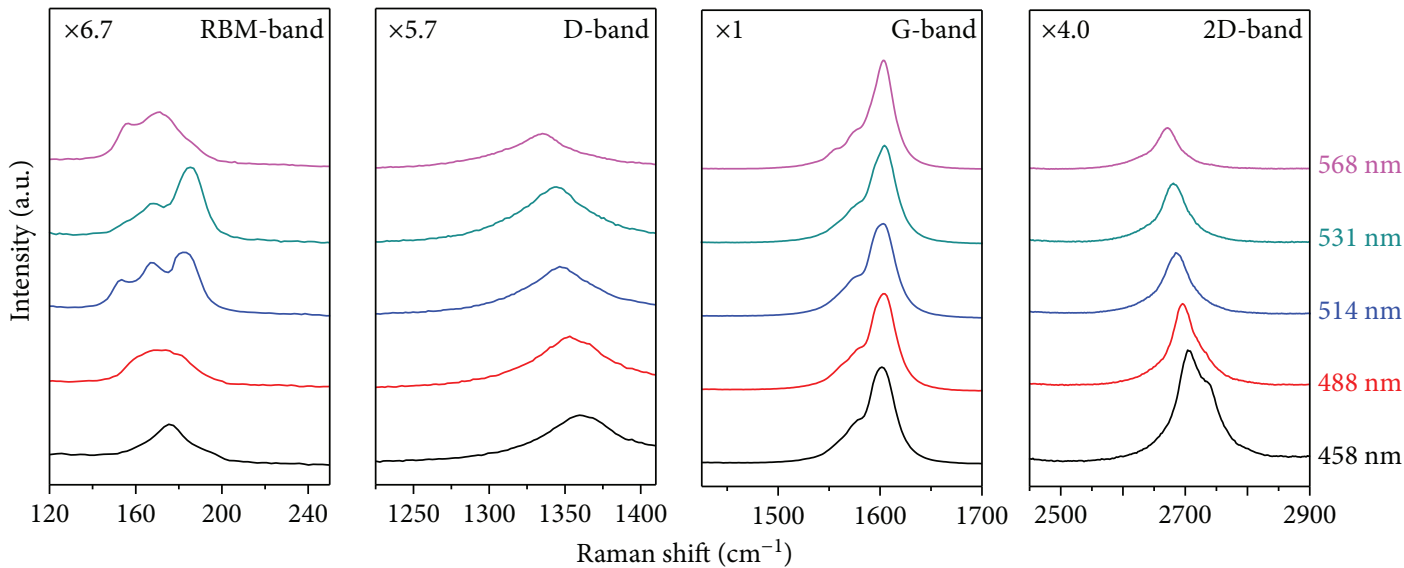

(b) AgCI@SWCNT

Figure 2: The RBM, D, G, and 2D-bands of Raman spectra of the pristine (a) and AgCl-filled (b) SWCNTs acquired at different laser wavelengths. The spectra were normalized to the area intensity of the G-band and offset for clarity.

Different lasers excite different electronic transitions in SWCNTs. Figure 3(a) shows the Kataura plot that relates the RBM frequency and optical transition energy of SWCNTs [28], where the laser wavelengths used for Raman spectroscopy and mean diameter of SWCNTs are denoted. As followed from the Kataura plot, the lasers with wavelengths of $458-568 \mathrm{~nm}$ excite the electronic transitions between the third and fourth van Hove singularities in valence band and conduction band of $1.4 \mathrm{~nm}$ mean diameter semiconducting SWCNTs.

To analyze the filling-induced alteration of the RBMband of SWCNTs, it was fitted with individual components. It is known that the position of peaks in the RBM-band of SWCNTs $\left(\omega_{\mathrm{RBM}}\right)$ is inversely proportional to the nanotube diameter $\left(d_{\mathrm{t}}\right)$ by the equation

$$
\omega_{\mathrm{RBM}}=\frac{227}{d_{\mathrm{t}}} \sqrt{1+C d_{\mathrm{t}}^{2}},
$$

where $C=0.05786 \mathrm{~nm}^{-2}$ [29]. Thus, the fitting of the RBMband allows analyzing the diameter distribution of nanotubes in the sample. Table 1 summarizes the results of the fitting of the RBM-band of the pristine and filled SWCNTs (positions of peak and their relative area intensities) together with nanotube diameters calculated using (1).

The RBM-band of Raman spectrum of the pristine SWCNTs obtained with $458 \mathrm{~nm}$ laser includes two peaks at 171 and $178 \mathrm{~cm}^{-1}$, which correspond to SWCNTs with diameters of 1.4 and $1.3 \mathrm{~nm}$, respectively. The RBM-band of the filled SWCNTs includes three peaks at 166, 177, and $190 \mathrm{~cm}^{-1}$, which correspond to nanotubes with diameters of $1.4,1.3$, and $1.2 \mathrm{~nm}$, accordingly (Figure 3(b)). In the spectra of both samples, the most intense component of the RBMband is positioned at $177-178 \mathrm{~cm}^{-1}$. The RBM-band of Raman spectrum of the pristine SWCNTs obtained at laser wavelength of $488 \mathrm{~nm}$ includes two peaks at 169 and $184 \mathrm{~cm}^{-1}$, which belong to SWCNTs with diameters of 1.4 and $1.3 \mathrm{~nm}$, respectively. The RBM-band of the filled SWCNTs includes four peaks at 159, 168, 180, and $180 \mathrm{~cm}^{-1}$, which correspond to nanotubes with diameters of $1.5,1.4,1.3$, and $1.2 \mathrm{~nm}$, accordingly (Figure $3(\mathrm{c})$ ). Therefore, the spectrum of the filled SWCNTs demonstrates the appearance of new peaks of larger and smaller diameter nanotubes.

At laser wavelength of $514 \mathrm{~nm}$, the RBM-band of the pristine SWCNTs includes five peaks at 157, 168, 178, 184, and $189 \mathrm{~cm}^{-1}$, which are assigned to nanotubes with diameters 


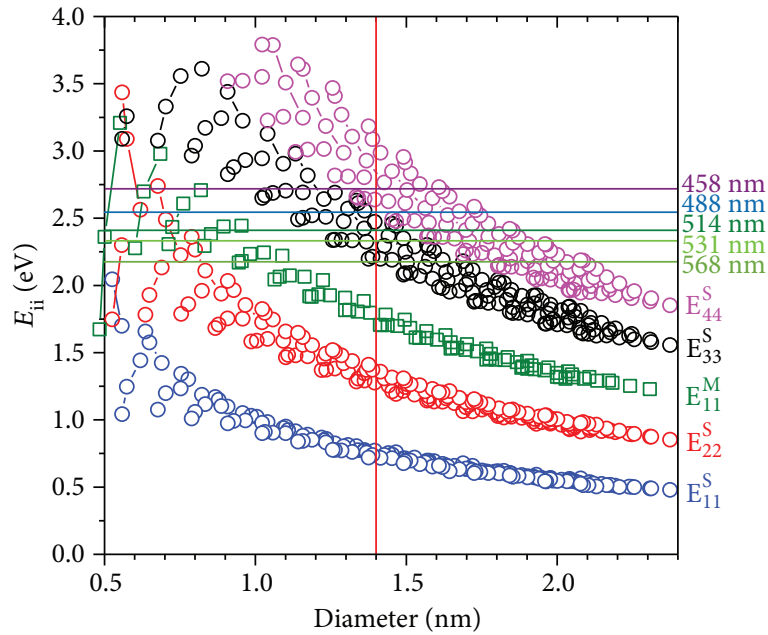

(a)

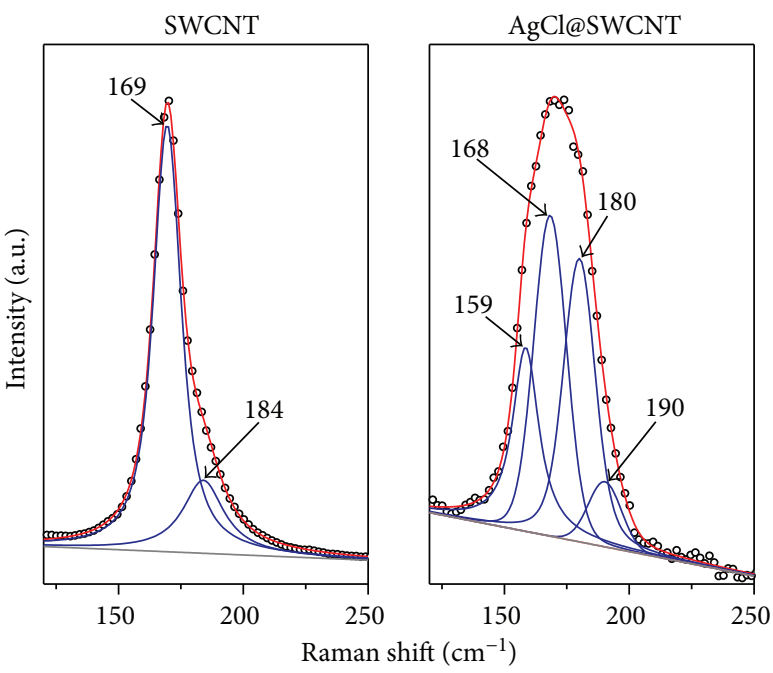

(c) $\lambda_{\text {ex }}=488 \mathrm{~nm}$

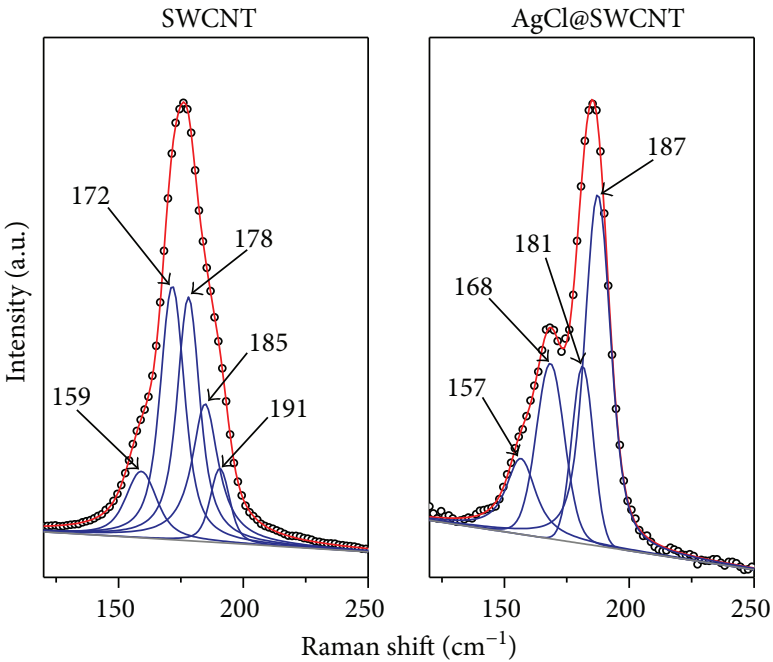

(e) $\lambda_{\mathrm{ex}}=531 \mathrm{~nm}$

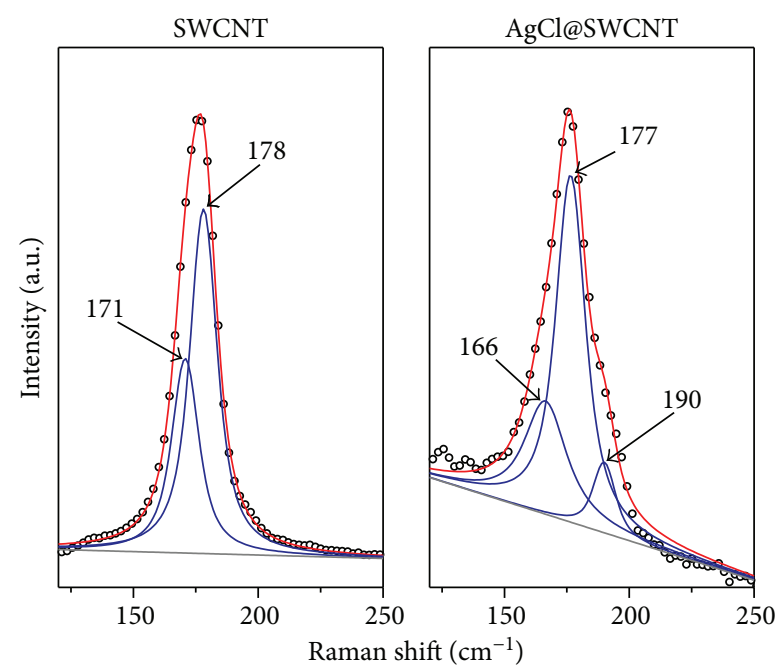

(b) $\lambda_{\mathrm{ex}}=458 \mathrm{~nm}$

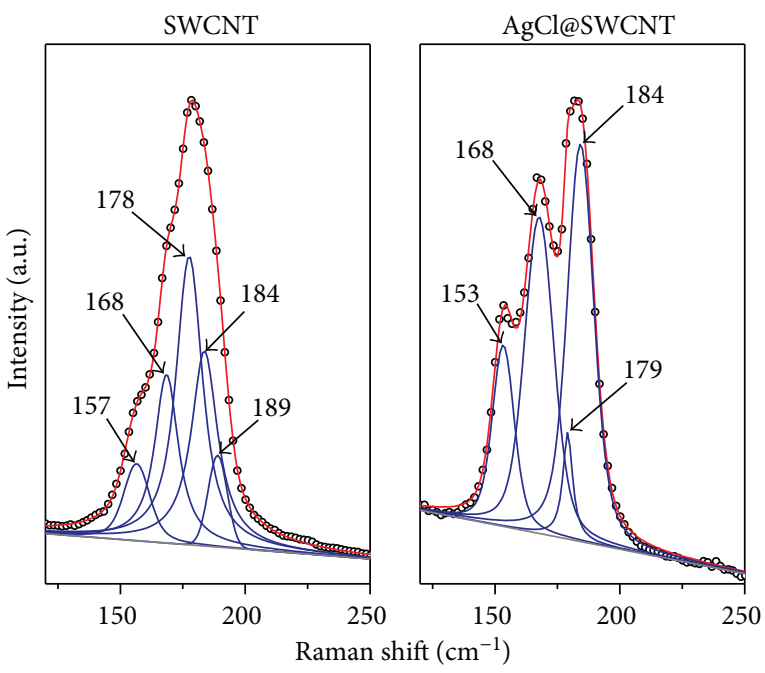

(d) $\lambda_{\text {ex }}=514 \mathrm{~nm}$

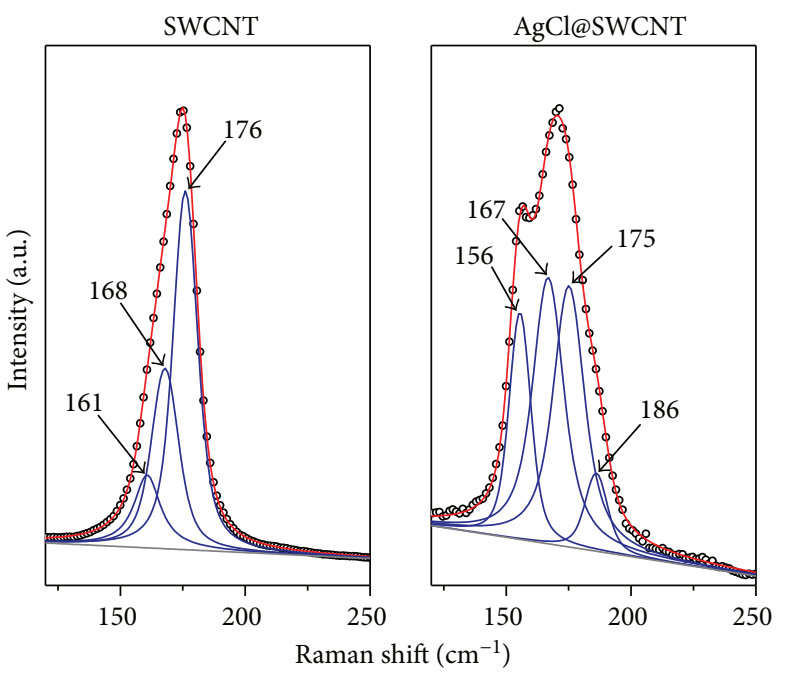

(f) $\lambda_{\mathrm{ex}}=568 \mathrm{~nm}$

Figure 3: (a) The Kataura plot (replotted from [28]), where the laser wavelengths used for Raman spectroscopy and mean diameter of SWCNTs are denoted. (b-f) The RBM-bands of Raman spectra of the pristine and AgCl-filled SWCNTs fitted with individual components. The positions of the maxima of components are indicated. 
TABLE 1: The peak positions (RS) and relative area intensity (I) of the individual components of the RBM-band of Raman spectra of the pristine and AgCl-filled SWCNTs as well as diameters of the corresponding nanotubes. Asterisks denote the peaks in the spectra of the filled SWCNTs that are the same as in the spectra of the pristine SWCNTs.

\begin{tabular}{|c|c|c|c|c|c|c|}
\hline \multirow{3}{*}{$\lambda_{\mathrm{ex}}(\mathrm{nm})$} & \multicolumn{3}{|c|}{ SWCNT } & \multicolumn{3}{|c|}{ AgCl@SWCNT } \\
\hline & \multicolumn{2}{|c|}{$\begin{array}{c}\text { Component of } \\
\text { RBM-band }\end{array}$} & \multirow[t]{2}{*}{ Tube diameter, $\mathrm{nm}$} & \multicolumn{2}{|c|}{$\begin{array}{l}\text { Component of } \\
\text { RBM-band }\end{array}$} & \multirow[t]{2}{*}{ Tube diameter, nm } \\
\hline & RS & I & & RS & I & \\
\hline \multirow{3}{*}{458} & 171 & 0.36 & 1.4 & 166 & 0.30 & 1.4 \\
\hline & 178 & 0.64 & 1.3 & $177^{*}$ & 0.61 & 1.3 \\
\hline & & & & 190 & 0.09 & 1.2 \\
\hline \multirow{4}{*}{488} & 169 & 0.78 & 1.4 & 159 & 0.23 & 1.5 \\
\hline & 184 & 0.22 & 1.3 & $168^{*}$ & 0.35 & 1.4 \\
\hline & & & & 180 & 0.33 & 1.3 \\
\hline & & & & 190 & 0.09 & 1.2 \\
\hline \multirow{5}{*}{514} & 157 & 0.08 & 1.5 & 153 & 0.16 & 1.6 \\
\hline & 168 & 0.20 & 1.4 & $168^{*}$ & 0.36 & 1.4 \\
\hline & 178 & 0.38 & 1.3 & $179^{*}$ & 0.05 & 1.3 \\
\hline & 184 & 0.27 & 1.3 & $184^{*}$ & 0.43 & 1.3 \\
\hline & 189 & 0.07 & 1.2 & & & \\
\hline \multirow{5}{*}{531} & 159 & 0.11 & 1.5 & $157^{*}$ & 0.14 & 1.5 \\
\hline & 172 & 0.31 & 1.4 & $168^{*}$ & 0.22 & 1.4 \\
\hline & 178 & 0.30 & 1.3 & $181^{*}$ & 0.16 & 1.3 \\
\hline & 185 & 0.21 & 1.3 & $187^{*}$ & 0.48 & 1.3 \\
\hline & 191 & 0.07 & 1.2 & & & \\
\hline \multirow{4}{*}{568} & 161 & 0.14 & 1.5 & 156 & 0.20 & 1.6 \\
\hline & 168 & 0.31 & 1.4 & $167^{*}$ & 0.37 & 1.4 \\
\hline & 176 & 0.55 & 1.4 & $175^{*}$ & 0.36 & 1.4 \\
\hline & & & & 186 & 0.07 & 1.3 \\
\hline
\end{tabular}

of $1.5,1.4,1.3$, and $1.2 \mathrm{~nm}$. The RBM-band of the filled nanotubes includes four peaks at 153,168,179, and $184 \mathrm{~cm}^{-1}$, which belong to $1.3-1.6 \mathrm{~nm}$ diameter SWCNTs (Figure 3(d)). The three last peaks are the same as in the spectrum of the pristine nanotubes. However, their relative intensity is changed. For the pristine SWCNTs, the peak at $178 \mathrm{~cm}^{-1}$ had the largest intensity, whereas for the filled SWCNTs, the peak at $184 \mathrm{~cm}^{-1}$ has the maximal intensity. At laser wavelength of $531 \mathrm{~nm}$, the spectrum of the pristine SWCNTs includes five peaks at 159, 172, 178, 185, and $191 \mathrm{~cm}^{-1}$, which correspond to nanotubes with diameters of $1.5,1.4,1.3$, and $1.2 \mathrm{~nm}$. All these peaks excluding the last one are present in the spectrum of the filled SWCNTs (Figure 3(e)). However, their relative intensities are altered. In the spectrum of the pristine nanotubes, the peaks at 172 and $178 \mathrm{~cm}^{-1}$ had the maximal intensity, whereas in the spectrum of the filled SWCNTs, the peak at $187 \mathrm{~cm}^{-1}$ has the largest intensity. At laser wavelength of $568 \mathrm{~nm}$, the RBM-band of the pristine SWCNTs includes three peaks at 161, 168, and $176 \mathrm{~cm}^{-1}$, which are assigned to nanotubes with diameters of 1.5 and $1.4 \mathrm{~nm}$. The spectrum of the filled SWCNTs includes fours peaks at $156,167,175$, and $186 \mathrm{~cm}^{-1}$, which belong to nanotubes with diameters of $1.6,1.4$, and $1.3 \mathrm{~nm}$ (Figure 3(f)). In the spectrum of the pristine SWCNTs, the peak at $176 \mathrm{~cm}^{-1}$ has the largest intensity, whereas in the case of the filled SWCNTs, the peaks at 167 and $175 \mathrm{~cm}^{-1}$ have the maximal intensity.

The above-described changes in the RBM-band of the filled SWCNTs as compared to the pristine SWCNTs that include changes in peak intensities and appearance of new peaks testify to filling-induced alteration of resonance excitation conditions of nanotubes. They are possibly a result of charge transfer in the filled nanotubes that causes exciting optical transitions in smaller or larger diameter nanotubes using the laser with the same wavelength. In the literature, there are no reports on a detailed analysis and comparison of the RBM-band of Raman spectra of the pristine semiconducting SWCNTs and filled nanotubes acquired with multifrequency Raman spectroscopy. In [24], the Raman spectra of $1.4 \mathrm{~nm}$ diameter metallicity-mixed SWCNTs filled with silver halogenides were acquired at three laser wavelengths (514, 633, and $785 \mathrm{~nm})$. According to the Kataura plot [28], only one of these wavelengths $(514 \mathrm{~nm})$ is suited for measurements of semiconducting SWCNTs. However, it was shown that the RBM-band of Raman spectra obtained at this laser wavelength stayed unchanged upon filling. In contrast, in the present work, we used 5 laser wavelengths to excite selectively semiconducting SWCNTs and observed the alteration of the RBM-bands of the filled SWCNTs. Thus, using multifrequency Raman spectroscopy allowed firstly 


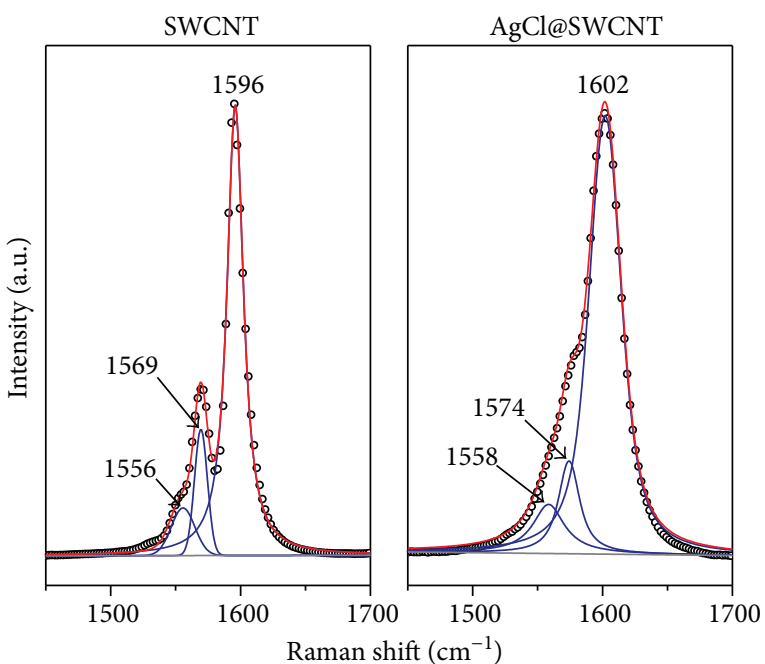

(a) $\lambda_{\mathrm{ex}}=458 \mathrm{~nm}$

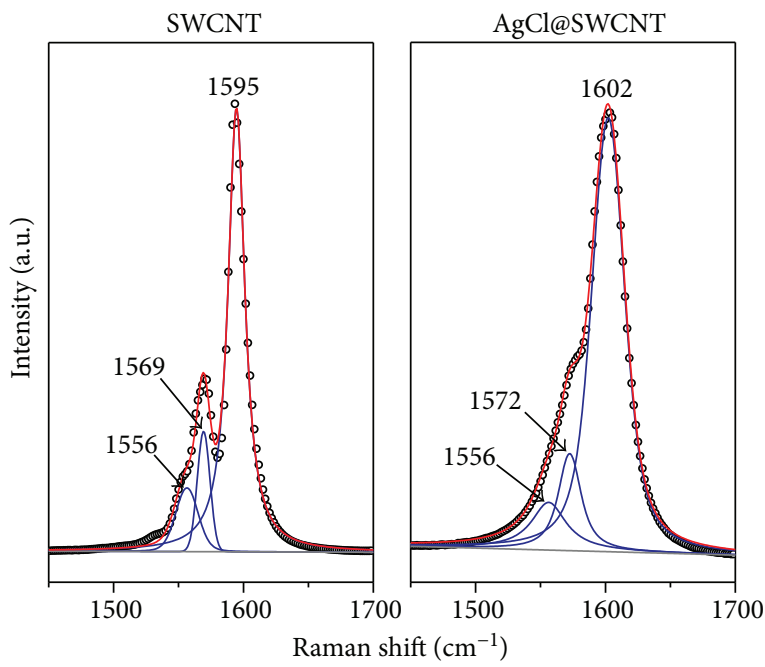

(c) $\lambda_{\mathrm{ex}}=514 \mathrm{~nm}$

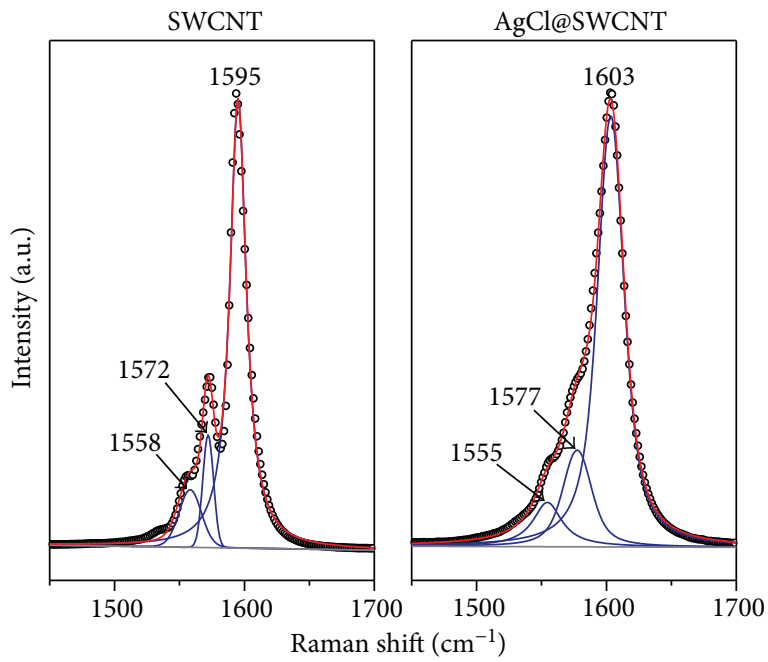

(e) $\lambda_{\text {ex }}=568 \mathrm{~nm}$

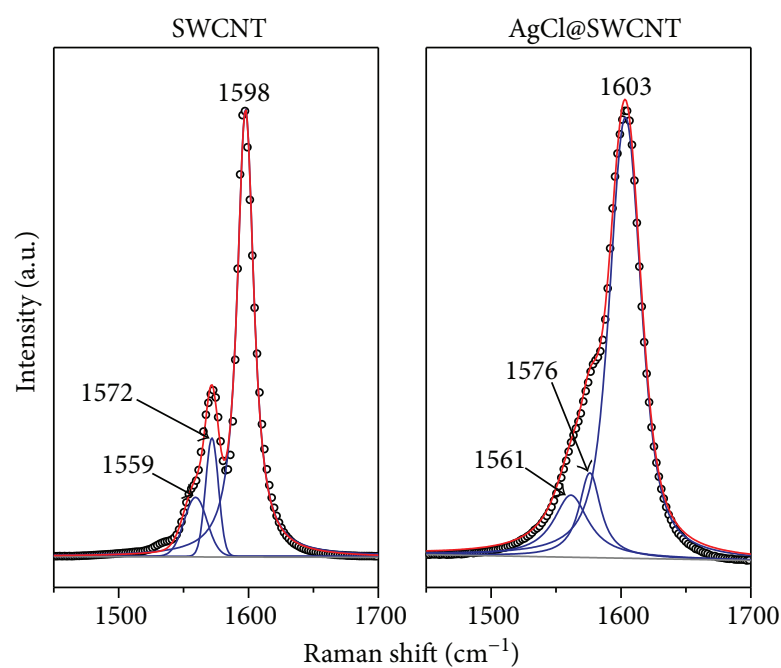

(b) $\lambda_{\mathrm{ex}}=488 \mathrm{~nm}$

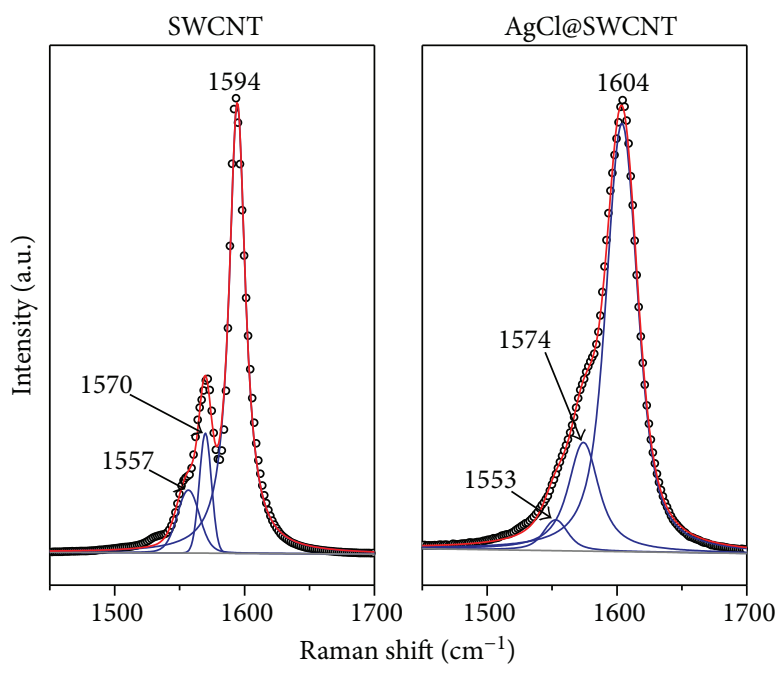

(d) $\lambda_{\mathrm{ex}}=531 \mathrm{~nm}$

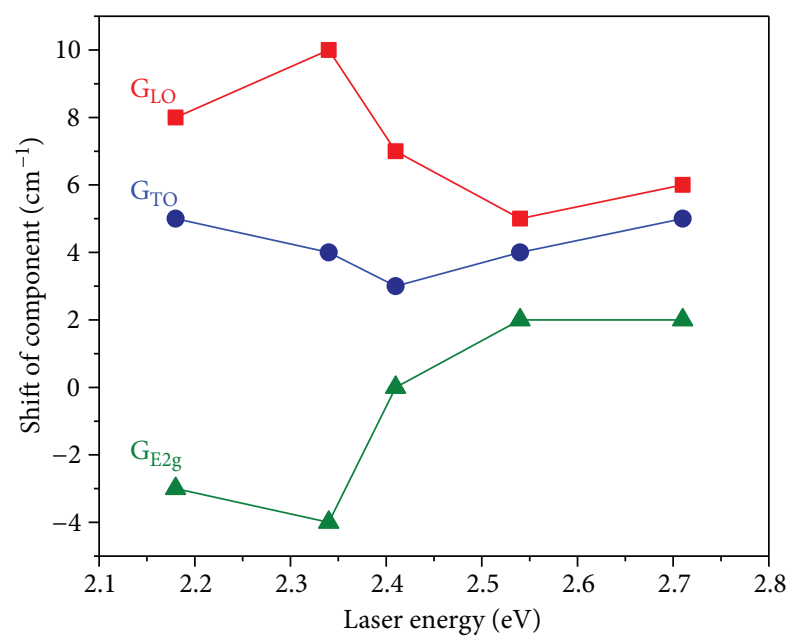

(f)

FIGURE 4: (a-e) The G-bands of Raman spectra of the pristine and AgCl-filled SWCNTs fitted with individual components $\mathrm{G}_{\mathrm{E} 2 \mathrm{~g}}, \mathrm{G}_{\mathrm{TO}}$, and $\mathrm{G}_{\mathrm{LO}}$. The positions of the maxima of components are denoted. (f) The shift of components of the G-band relative to the positions of the pristine SWCNTs plotted versus laser energy. 
TABLE 2: The peak positions (RS) and relative area intensity (I) of the components of the G-band $\left(\mathrm{G}_{\mathrm{E} 2 \mathrm{~g}}\right.$, $\mathrm{G}_{\mathrm{TO}}$, and $\left.\mathrm{G}_{\mathrm{LO}}\right)$ of Raman spectra of the pristine and AgCl-filled SWCNTs. The shifts of the components of the filled SWCNTs relative to the position of the pristine nanotubes are given in parentheses.

\begin{tabular}{lccccccc}
\hline \multirow{2}{*}{$\lambda_{\text {ex }}(\mathrm{nm})$} & \multicolumn{2}{c}{$\mathrm{G}_{\mathrm{E} 2 \mathrm{~g}}$} & \multicolumn{3}{c}{ Component of G-band $\left(\mathrm{cm}^{-1}\right)$} & \multicolumn{2}{c}{$\mathrm{G}_{\mathrm{LO}}$} \\
& Sample & $\mathrm{RS}$ & $\mathrm{I}$ & $\mathrm{RS}$ & $\mathrm{G}$ & $\mathrm{RS}$ & $\mathrm{I}$ \\
\hline \multirow{2}{*}{458} & SWCNT & 1556 & 0.08 & 1569 & 0.12 & 1596 & 0.80 \\
& AgCl@SWCNT & $1558(+2)$ & 0.10 & $1574(+5)$ & 0.12 & $1602(+6)$ & 0.78 \\
\multirow{4}{*}{488} & SWCNT & 1559 & 0.10 & 1572 & 0.11 & 1598 & 0.79 \\
& AgCl@SWCNT & $1561(+2)$ & 0.14 & $1576(+4)$ & 0.11 & $1603(+5)$ & 0.75 \\
514 & SWCNT & 1556 & 0.11 & 1569 & 0.11 & 1595 & 0.78 \\
& AgCl@SWCNT & 1556 & 0.11 & $1572(+3)$ & 0.13 & $1602(+7)$ & 0.76 \\
531 & SWCNT & 1557 & 0.11 & 1570 & 0.10 & 1594 & 0.79 \\
& AgCl@SWCNT & $1553(-4)$ & 0.04 & $1574(+4)$ & 0.20 & $1604(+10)$ & 0.76 \\
568 & SWCNT & 1558 & 0.10 & 1572 & 0.09 & 1595 & 0.81 \\
& AgCl@SWCNT & $1555(-3)$ & 0.09 & $1577(+5)$ & 0.17 & $1603(+8)$ & 0.74 \\
\hline
\end{tabular}

detecting the charge transfer-induced changes in resonance conditions of 1.2-1.6 nm diameter semiconducting SWCNTs upon their filling.

Now we turn to the analysis of the G-band of Raman spectra of the pristine and AgCl-filled SWCNTs acquired at laser wavelengths of $458-568 \mathrm{~nm}$. Figure 4 shows the Gbands fitted with three individual components $G_{\mathrm{E} 2 \mathrm{~g}}, \mathrm{G}_{\mathrm{TO}}$, and $\mathrm{G}_{\mathrm{LO}}$. The components $\mathrm{G}_{\mathrm{LO}}$ and $\mathrm{G}_{\mathrm{TO}}$ stem from longitudinal optical (LO) and transversal optical (TO) phonons, respectively [30]. The component $\mathrm{G}_{\mathrm{E} 2 \mathrm{~g}}$ appears due to the resonant $\mathrm{E}_{2 \mathrm{~g}}$ mode in semiconducting SWCNTs [20]. Table 2 summarizes the results of the fitting of the G-band of the pristine and filled SWCNTs.

The G-band of Raman spectra of the pristine SWCNTs acquired at laser wavelength of $458 \mathrm{~nm}$ includes three components at 1556,1569 , and $1596 \mathrm{~cm}^{-1}$. In the spectrum of the filled SWCNTs, the components are upshifted by $2-6 \mathrm{~cm}^{-1}$ and positioned at 1558,1574 , and $1602 \mathrm{~cm}^{-1}$. Their relative intensities stay unchanged upon filling (Figure 4(a)). The same trends (an upshift and unchanged relative intensity of components) are observed in the G-band of Raman spectra of filled nanotubes obtained at other laser wavelengths (Figures 4(b)-4(e)). It should be noted that the G-band of Raman spectra of the filled SWCNTs acquired at all laser wavelengths has a narrow Lorentzian shape, which is a characteristic of semiconducting SWCNTs [20]. The semiconducting profile of the G-band of the filled nanotubes testifies that SWCNTs remain in a semiconducting state upon filling.

To compare the shifts of the components of the G-band of Raman spectra obtained with different lasers, they were plotted versus laser energy in Figure 4(f). It is visible that the shifts of the $\mathrm{G}_{\mathrm{LO}}$ component in the spectra obtained with different lasers amount to $5-10 \mathrm{~cm}^{-1}$. The shifts of the $\mathrm{G}_{\text {TO }}$ component are equal to $3-5 \mathrm{~cm}^{-1}$. These data show that the shifts of the $\mathrm{G}_{\mathrm{LO}}$ and $\mathrm{G}_{\mathrm{TO}}$ components are independent on used laser energy. The shift of the $\mathrm{G}_{\mathrm{E} 2 \mathrm{~g}}$ component ranges from -4 to $2 \mathrm{~cm}^{-1}$ at different laser energies.

The shifts of the components of the G-band may testify to doping-induced charge transfer in the filled SWCNTs.
Indeed, a similar upshift of the $\mathrm{G}_{\mathrm{LO}}$ and $\mathrm{G}_{\mathrm{TO}}$ components was observed for SWCNTs filled with metal halogenides and chalcogenides in [22-24]. This shift was generally attributed to $p$-doping of SWCNTs by encapsulated compounds. In particular, in [24], the upshift of the G-band of Raman spectra of metallicity-mixed SWCNTs filled with silver halogenides acquired at three laser wavelengths of 514, 633, and $785 \mathrm{~nm}$ was assigned to acceptor behavior of the compounds with the corresponding charge transfer from SWCNTs to the inserted salts. However, in this work, the detailed analysis of the G-bands of Raman spectra was not conducted. Moreover, in the present work, we use more laser wavelengths that excite selectively semiconducting SWCNTs that allows us to trace changes in the electronic properties of different semiconducting SWCNTs. We firstly investigate the dependence of shifts of the components of the G-band on laser wavelength. The obtained information about the modified electronic properties of filled purely semiconducting SWCNTs is important for their applications in nanoelectronics.

While the RBM- and G-bands of Raman spectra are significantly modified upon filling of SWCNTs with $\mathrm{AgCl}$, as it was discussed above, the $\mathrm{D}$ and 2D-bands are not affected by filling. The D and 2D-bands of Raman spectra of the pristine and filled SWCNTs demonstrate shifts of the peak positions toward lower frequencies with increasing laser wavelengths (Figure 2). Figure 5 presents the peak positions of the D and 2D-bands of Raman spectra of the pristine and $\mathrm{AgCl}$-filled SWCNTs plotted versus laser energy. The experimental data are shown together with their linear fits. These data show a high linearity. The slopes are $34 \pm 4 \mathrm{~cm}^{-1} \mathrm{eV}^{-1}$ and $85 \pm 7 \mathrm{~cm}^{-1} \mathrm{eV}^{-1}$ for SWCNTs, as well as $45 \pm 3 \mathrm{~cm}^{-1}$ $\mathrm{eV}^{-1}$ and $65 \pm 2 \mathrm{~cm}^{-1} \mathrm{eV}^{-1}$ for AgCl-filled SWCNTs. This testifies to high purity and quality of the pristine and filled nanotube samples.

\section{Conclusions}

In the present work, we filled the channels of semiconducting SWCNTs with silver chloride. The filling was confirmed by 


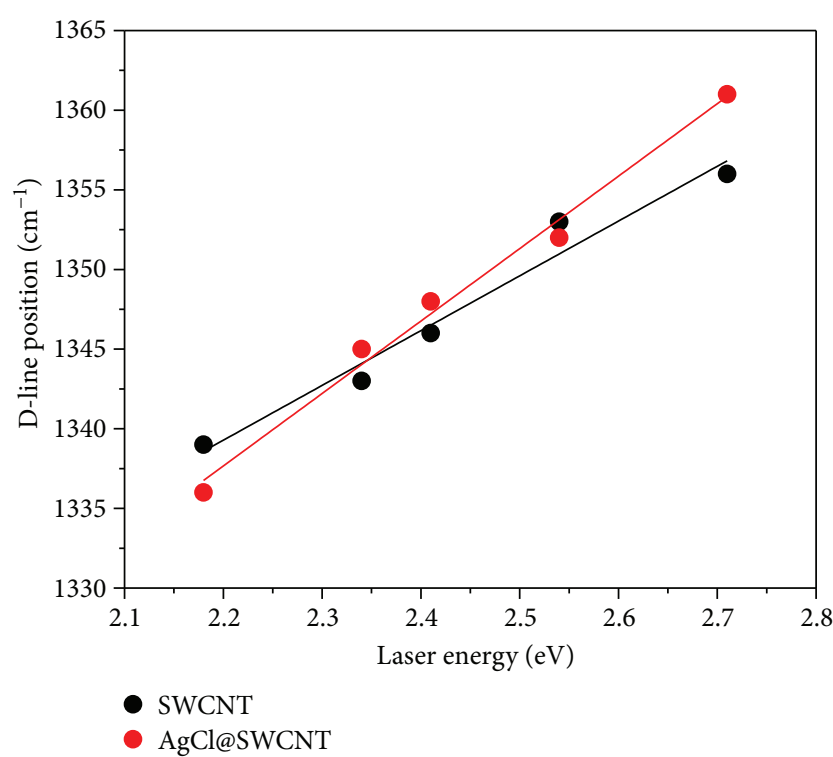

(a)

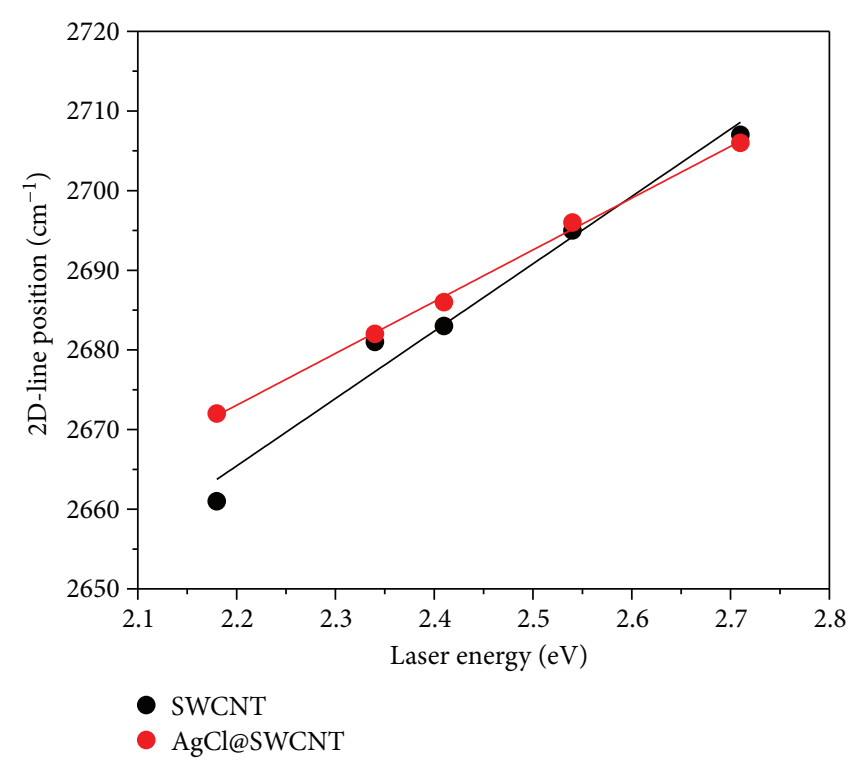

(b)

FIgURe 5: The positions of the D (a) and 2D (b) lines of Raman spectra of the pristine and AgCl-filled SWCNTs plotted versus laser energy. The experimental data are shown together with their linear fits.

HR STEM. The filling-induced modifications of Raman modes of SWCNTs were analyzed using five different laser wavelengths. The fitting of the RBM and G-bands of Raman spectra of the pristine and filled nanotubes with individual components allowed analyzing in detail the influence of encapsulated silver chloride on the electronic properties of different diameter nanotubes. The analysis of the RBMband allowed revealing the alteration of relative intensities of the individual components as well as the appearance of new peaks of smaller and larger diameter nanotubes. This was caused by changes in resonance excitation conditions of SWCNTs upon filling. The analysis of the G-band allowed revealing significant shifts of components and no changes in the component intensities, that is, keeping a semiconducting profile of the band. This was attributed to $p$-doping of nanotubes by incorporated silver chloride accompanied by charge transfer from nanotubes to the inserted salt.

\section{Conflicts of Interest}

The authors declare that there is no conflict of interests regarding the publication of this paper.

\section{Acknowledgments}

K. Yanagi acknowledges JSPS KAKENHI Grant no. JP16H00919.

\section{References}

[1] V. Meunier, A. G. Souza Filho, E. B. Barros, and M. S. Dresselhaus, "Physical properties of low-dimensional $s p^{2}$ based carbon nanostructures," Reviews of Modern Physics, vol. 88 , no. 2 , article $025005,2016$.
[2] B. Liu, F. Wu, H. Gui, M. Zheng, and C. Zhou, "Chirality-controlled synthesis and applications of single-wall carbon nanotubes," ACS Nano, vol. 11, no. 1, pp. 31-53, 2017.

[3] M. V. Kharlamova, "Advances in tailoring the electronic properties of single-walled carbon nanotubes," Progress in Materials Science, vol. 77, pp. 125-211, 2016.

[4] F. Yang, X. Wang, M. Li et al., "Templated synthesis of singlewalled carbon nanotubes with specific structure," Accounts of Chemical Research, vol. 49, no. 4, pp. 606-615, 2016.

[5] A. A. Tonkikh, V. I. Tsebro, E. A. Obraztsova et al., "Metallization of single-wall carbon nanotube thin films induced by gas phase iodination," Carbon, vol. 94, pp. 768-774, 2015.

[6] T. Fujimori, A. Morelos-Gómez, Z. Zhu et al., "Conducting linear chains of sulphur inside carbon nanotubes," Nature Communications, vol. 4, article 2162, 2013.

[7] T. Zoberbier, T. W. Chamberlain, J. Biskupek et al., "Investigation of the interactions and bonding between carbon and group VIII metals at the atomic scale," Small, vol. 12, no. 12, pp. 1649-1657, 2016.

[8] A. Botos, J. Biskupek, T. W. Chamberlain et al., "Carbon nanotubes as electrically active nanoreactors for multi-step inorganic synthesis: sequential transformations of molecules to nanoclusters and nanoclusters to nanoribbons," Journal of the American Chemical Society, vol. 138, no. 26, pp. 8175$8183,2016$.

[9] A. A. Eliseev, N. S. Falaleev, N. I. Verbitskiy et al., "Size-dependent structure relations between nanotubes and encapsulated nanocrystals," Nano Letters, vol. 17, no. 2, pp. 805-810, 2017.

[10] N. S. Falaleev, A. S. Kumskov, V. G. Zhigalina et al., "Capsulate structure effect on SWNTs doping in $\mathrm{Rb}_{\mathrm{x}} \mathrm{Ag}_{1-\mathrm{x}} \mathrm{I} @ \mathrm{SWNT}$ composites," CrystEngComm, vol. 19, no. 22, pp. 3063-3070, 2017.

[11] C. T. Stoppiello, J. Biskupek, Z. Y. Li et al., "A one-pot-onereactant synthesis of platinum compounds at the nanoscale," Nanoscale, vol. 9, no. 38, pp. 14385-14394, 2017.

[12] M. Kierkowicz, J. M. González-Domínguez, E. Pach et al., "Filling single-walled carbon nanotubes with lutetium 
chloride: a sustainable production of nanocapsules free of nonencapsulated material," ACS Sustainable Chemistry \& Engineering, vol. 5, no. 3, pp. 2501-2508, 2017.

[13] Y. V. Fedoseeva, A. S. Orekhov, G. N. Chekhova et al., "Singlewalled carbon nanotube reactor for redox transformation of mercury dichloride," ACS Nano, vol. 11, no. 9, pp. 86438649, 2017.

[14] J. H. Spencer, J. M. Nesbitt, H. Trewhitt et al., "Raman spectroscopy of optical transitions and vibrational energies of $\sim 1 \mathrm{~nm} \mathrm{HgTe}$ extreme nanowires within single walled carbon nanotubes," ACS Nano, vol. 8, no. 9, pp. 9044-9052, 2014.

[15] R. L. McSweeney, T. W. Chamberlain, M. Baldoni et al., "Direct measurement of electron transfer in nanoscale hostguest systems: metallocenes in carbon nanotubes," Chemistry A European Journal, vol. 22, no. 38, pp. 13540-13549, 2016.

[16] A. A. Tonkikh, D. V. Rybkovskiy, A. S. Orekhov et al., "Optical properties and charge transfer effects in single-walled carbon nanotubes filled with functionalized adamantane molecules," Carbon, vol. 109, pp. 87-97, 2016.

[17] B. Botka, M. E. Füstös, H. M. Tóháti et al., "Interactions and chemical transformations of coronene inside and outside carbon nanotubes,” Small, vol. 10, no. 7, pp. 1369-1378, 2014.

[18] J. Campo, Y. Piao, S. Lam et al., "Enhancing single-wall carbon nanotube properties through controlled endohedral filling," Nanoscale Horizons, vol. 1, no. 4, pp. 317-324, 2016.

[19] A. S. Sinitsa, T. W. Chamberlain, T. Zoberbier et al., "Formation of nickel clusters wrapped in carbon cages: toward new endohedral metallofullerene synthesis," Nano Letters, vol. 17, no. 2, pp. 1082-1089, 2017.

[20] R. Saito, M. Hofmann, G. Dresselhaus, A. Jorio, and M. S. Dresselhaus, "Raman spectroscopy of graphene and carbon nanotubes," Advances in Physics, vol. 60, no. 3, pp. 413-550, 2011.

[21] A. Jorio and A. G. Souza Filho, "Raman studies of carbon nanostructures," Annual Review of Materials Research, vol. 46, no. 1, pp. 357-382, 2016.

[22] M. V. Kharlamova, L. V. Yashina, and A. V. Lukashin, "Charge transfer in single-walled carbon nanotubes filled with cadmium halogenides," Journal of Materials Science, vol. 48, no. 24, pp. 8412-8419, 2013.

[23] J. H. Spencer, D. C. Smith, L. P. McDonnell, J. Sloan, and R. J. Kashtiban, "Coherence lifetime broadened optical transitions in a 2 atom diameter $\mathrm{HgTe}$ nanowire: a temperature dependent resonance Raman study," RSC Advances, vol. 6, no. 98, pp. 95387-95395, 2016.

[24] A. A. Eliseev, L. V. Yashina, M. M. Brzhezinskaya et al., "Structure and electronic properties of $\mathrm{AgX}(\mathrm{X}=\mathrm{Cl}, \mathrm{Br}, \mathrm{I})$ intercalated single-walled carbon nanotubes," Carbon, vol. 48, no. 10, pp. 2708-2721, 2010.

[25] K. Yanagi, H. Udoguchi, S. Sagitani et al., "Transport mechanisms in metallic and semiconducting single-wall carbon nanotube networks," ACS Nano, vol. 4, no. 7, pp. 4027-4032, 2010.

[26] M. V. Kharlamova, C. Kramberger, M. Sauer, K. Yanagi, and T. Pichler, "Comprehensive spectroscopic characterization of high purity metallicity-sorted single-walled carbon nanotubes," Physica Status Solidi B, vol. 252, no. 11, pp. 2512-2518, 2015.

[27] F. Kirchhoff, J. M. Holender, and M. J. Gillan, "Energetics and electronic structure of silver chloride," Physical Review B, vol. 49, no. 24, pp. 17420-17423, 1994.
[28] H. Kataura, Y. Kumazawa, Y. Maniwa et al., "Optical properties of single-wall carbon nanotubes," Synthetic Metals, vol. 103, no. 1-3, pp. 2555-2558, 1999.

[29] P. T. Araujo, I. O. Maciel, P. B. C. Pesce et al., "Nature of the constant factor in the relation between radial breathing mode frequency and tube diameter for single-wall carbon nanotubes," Physical Review B, vol. 77, no. 24, article 241403, 2008.

[30] M. Fouquet, H. Telg, J. Maultzsch et al., "Longitudinal optical phonons in metallic and semiconducting carbon nanotubes," Physical Review Letters, vol. 102, no. 7, article 075501, 2009. 

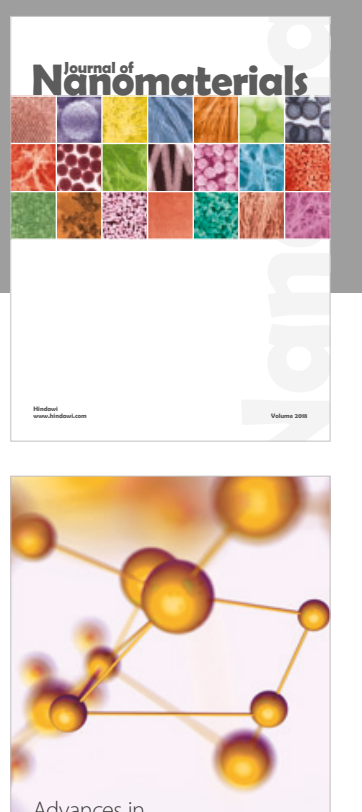

Physical Chemistry
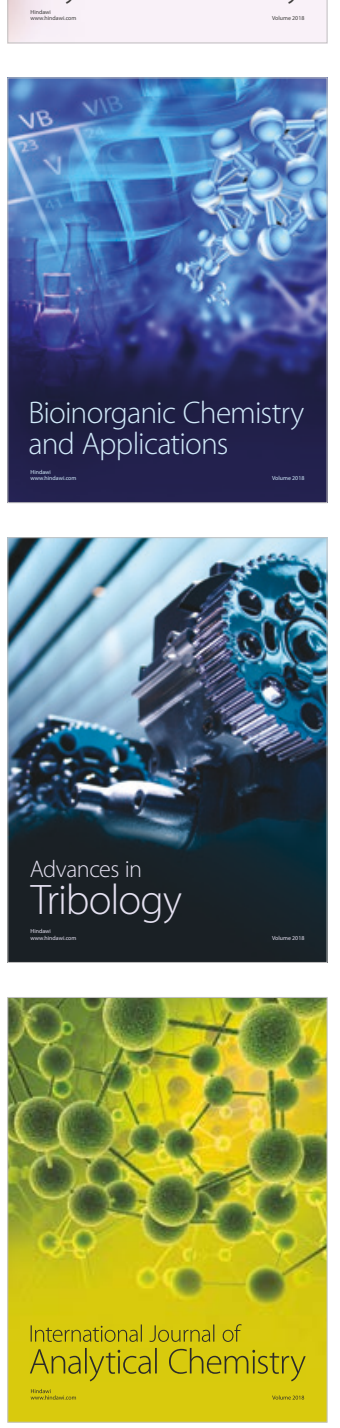

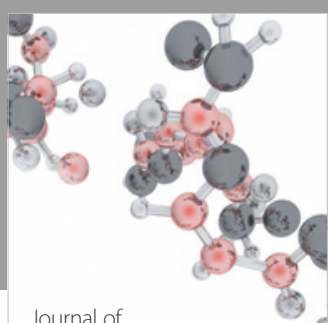

Analytical Methods

in Chemistry

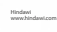

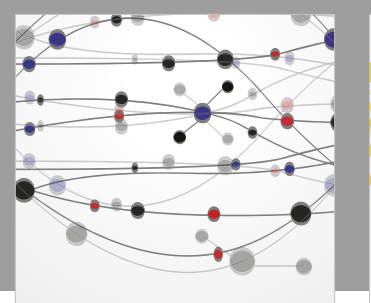

The Scientific World Journal

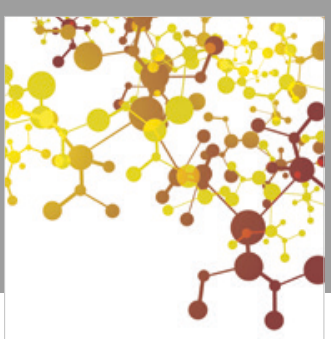

Journal of

Applied Chemistry
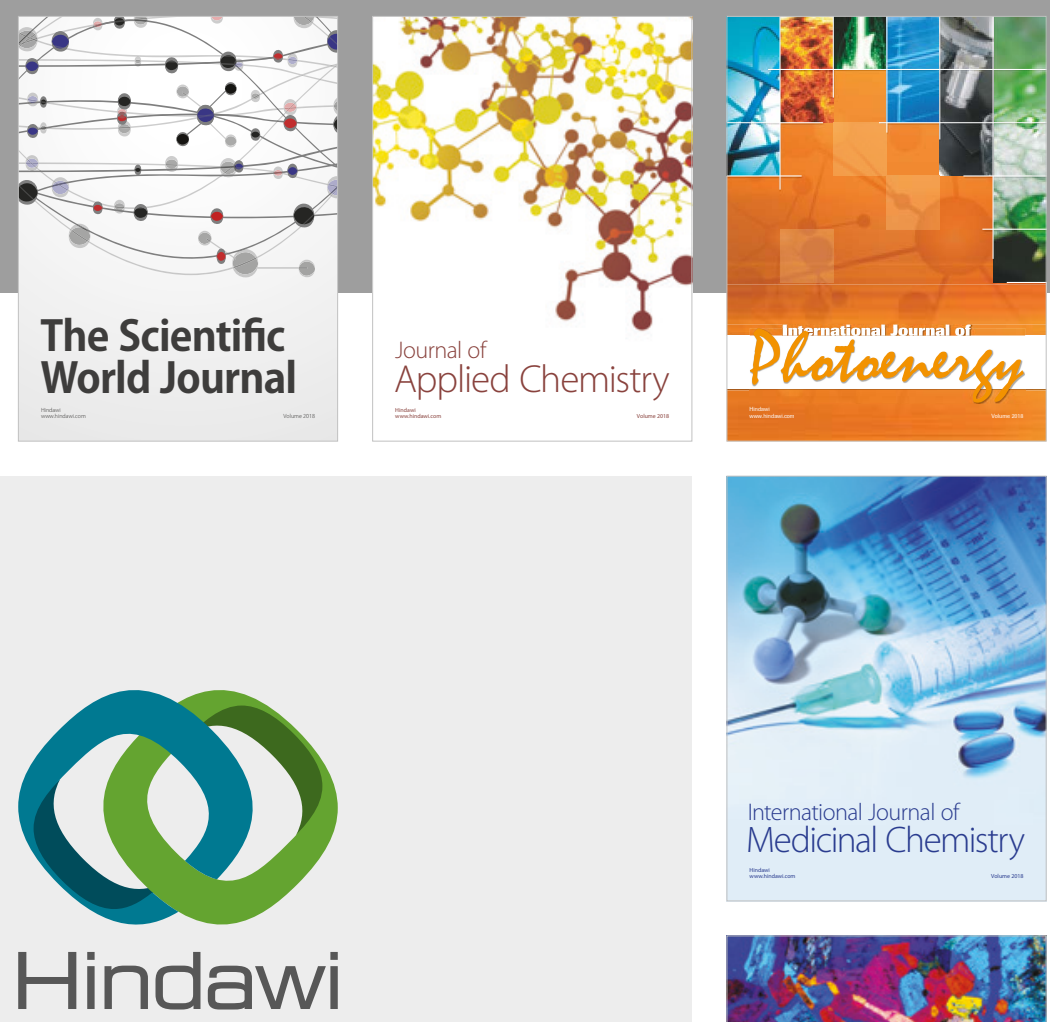

Submit your manuscripts at

www.hindawi.com
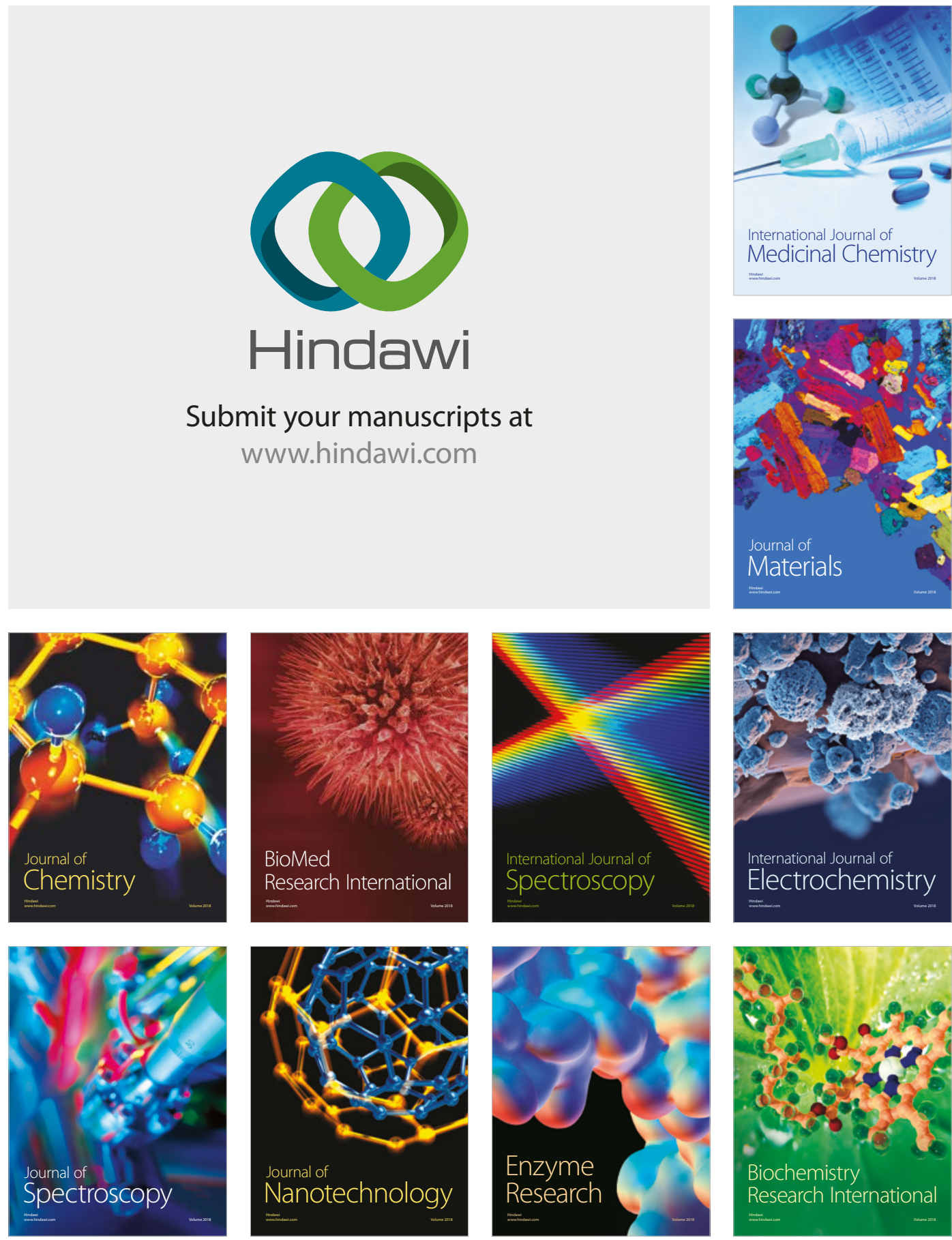
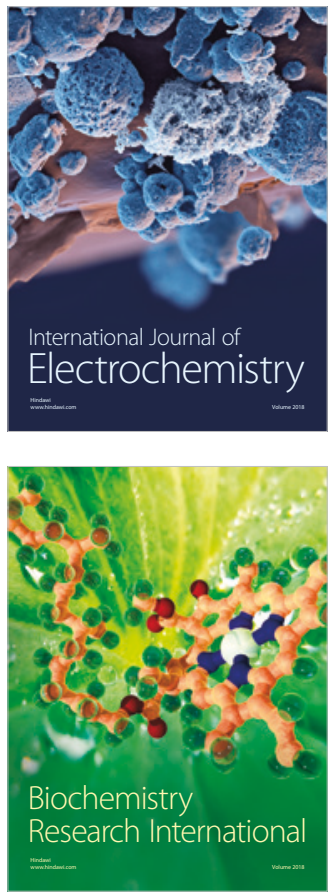